\title{
Design of a compact objective for SWIR applications
}

\author{
Jose M. Infante Herrero ${ }^{1,3}$, Marta C. de la Fuente ${ }^{3}$, Wang Lin ${ }^{1}$, Juan Carlos Miñano Dominguez ${ }^{1}$, Pablo \\ Benítez $^{1}$; Guillermo Biot ${ }^{1}$, Hammed Ahmadpanahi ${ }^{1}$; Andres F. Cifuentes ${ }^{4}$ \\ ${ }^{1}$ Universidad Politécnica de Madrid, CeDInt, Campus Montegancedo, 28223 Madrid, Spain, ${ }^{2}$ Light Prescriptions Innovators-Europe, Edif. \\ CeDInt Campus Montegancedo UPM, 28223 Pozuelo, Madrid, Spain, ${ }^{3}$ Indra Sistemas SA, Joaquín Rodrigo 11, 28300 Aranjuez, \\ Spain.,SECPhO (Spain) \\ jose.infante@cedint.upm.es
}

\begin{abstract}
Lately the short-wave infrared (SWIR) has become very important due to the recent appearance on the market of the small detectors with a large focal plane array. Military applications for SWIR cameras include handheld and airborne systems with long range detection requirements, but where volume and weight restrictions must be considered. In this paper we present three different designs of telephoto objectives that have been designed according to three different methods. Firstly the conventional method where the starting point of the design is an existing design. Secondly we will face design starting from the design of an aplanatic system. And finally the simultaneous multiple surfaces (SMS) method, where the starting point is the input wavefronts that we choose. The designs are compared in terms of optical performance, volume, weight and manufacturability. Because the objectives have been designed for the SWIR waveband, the color correction has important implications in the choice of glass that will be discussed in detail
\end{abstract}

Keywords: SWIR cameras, aplanatic system, telephoto, SMS design

\section{Introduction}

There is a boom in short-wave infrared (SWIR) systems for defense applications due to the development of small-size sensors for this band. This band allows the user to detect laser designators operating at $1060 \mathrm{~nm}$ and $1550 \mathrm{~nm}$, besides this band is very suitable to see through the smoke and detect camouflaged targets. For all these reasons there is a big interest in design light and small SWIR cameras for UAVs, handheld cameras and security systems.

At the same time that the technology of the detectors is developing fast, the optics for these detectors must be more compact and fulfill the user requirements for minimum dimensions.

In this study we undertake the design of an objective optimized for use in the SWIR band. The goal of our study is to present different techniques to face the problem of designing a spotter lens with the minimum size and volume. Basically these kinds of constraints can be reached during the optimization process. The glass choice is another issue that must be considered during the optimization process, as in the SWIR band the numbers of pairs for doing doublets is less than in the visible range. For the glass selection we have used a macro provided by CODEV ${ }^{\circledR}$ in order to do the optimization process more efficiently.

\section{Establishment of the requirements for the system}

The first step is to establish the requirements for the objective. Although the purpose of this paper is not to present a final design which would include the interface with the detector, the detector size brings us to the system focal length for a certain FOV. However the f/\# of the systems should be considered in a different way due to the fact that the relation between the pixel size and the $\mathrm{f} / \#$ lead you to obtain the desired SNR for the application.

For our study we are going to design an objective for a SWIR detector of 640 x 512 pixels and 20 microns pitch size. The field of view shall be $0.92^{\circ} \times 0.73^{\circ}$. Taking into account the detector size and field of view, the effective focal length shall be $800 \mathrm{~mm}$. Considering that we have a large value for the focal length we should take care in the choice of the $\mathrm{f} / \#$ as it will drive EPD and thus volume. Obviously, in order to have a small design in terms of volume, you need to design a slow system. But the question is: how slow can your system be in order to meet the range performance requirements for your application? We followed the criteria described in [1] and [2] that is based on the optical $Q$ that is a convenient and simple way of describing the theoretical limit 
of an imaging system, and it is defined as the relationship between the optical frequency cut-off and detector Nyquist frequency:

$$
Q=\frac{\lambda f / \#}{p}
$$

Where $\lambda$ is the wavelength, $\mathrm{f} \#$ is the $\mathrm{f}$-number and $p$ is the detector pitch. If we consider that the fill factor for the pixel it is nearly $100 \%$ then $p \approx x_{\text {det }}$. The Nyquist frequency $f_{N}$ for a focal plane array with a detector pitch of $x_{\text {det }}$ is given by

$$
f_{N}=\frac{1}{2 x_{\mathrm{det}}}
$$

The optical system cut-off frequency is given by

$$
f_{c o}=\frac{1}{\lambda f \#}
$$

If we use (2) and (3) we obtain the most common expression of $Q$ as

$$
Q=\frac{2 f_{N}}{f_{c o}}
$$

Thus, for efl $=800 \mathrm{~mm}$ and $\mathrm{f} / 8$ we obtain for our application a value of $\mathrm{Q}=0.52$ and a reasonable size for the entrance pupil diameter.

Finally the optics has to match the detector spectral response that spans from $900 \mathrm{~nm}$ to $1700 \mathrm{~nm}$.

\section{Facing the optical design}

Once the optical designer has all the requirements for the system he must decide how to start the design. Although the optimization process for the designs is inevitable there are different ways to define the starting point that should, wherever possible, be a configuration which is inherently capable of meeting the specification for the design.

In this work we are going to present three different approaches, each from different starting points.

\subsection{Using an existing design}

Since we want to achieve long focal length but short total length, a telephoto lens must be designed and a catadrioptic configuration is always a good choice. For the first approach we will design the system starting from a conventional catadioptric objective that was originally used for a night vision system, and with all spherical surfaces. Original parameters are EFL $125 \mathrm{~mm}$ and OAL $90 \mathrm{~mm}$, that is, a 0.72 telephoto ratio. This objective was designed for a spectral waveband 480 to $900 \mathrm{~nm}$ and $\pm 4^{\circ}$ FOV. It is faster than the current requirement $\mathrm{f} / 1.47$, but EPD has a similar value to our current specification. So it seems a good candidate for our starting point. 


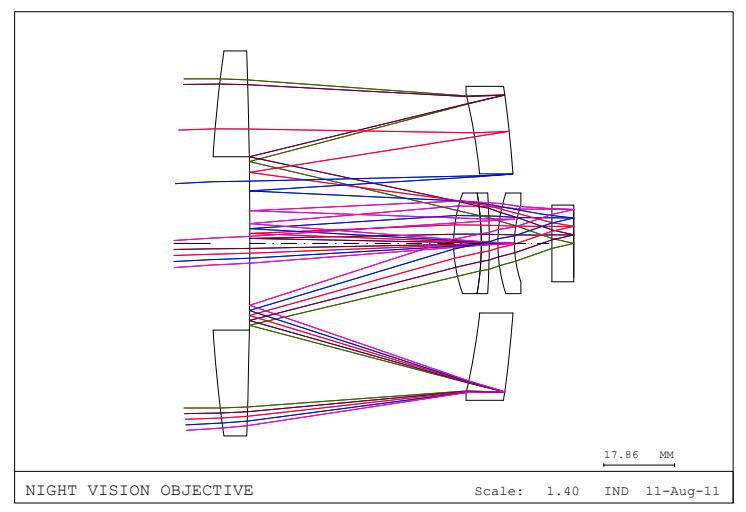

Figure 1: Conventional night vision objective f/1.47, efl 125, FOV $\pm 4^{\circ}$, oal $90 \mathrm{~mm}$.

With the current efl specification, maintaining the telephoto ratio would yield an unacceptable length so we have to increase focal length and telephoto ratio at the same time. On the contrary we have to reduce the obstruction ratio: the original one was close to $50 \%$, which was acceptable for an $f / 1.47$ system but it is not for an $f / 8$. As a result we need to increase power on the first lens and the first mirror. As this will make tolerances tighter, we define several zoom positions with decentered elements that are optimized simultaneously.

This design uses the first lens second surface as second mirror, which is an elegant solution from the manufacturability point of view. But since the first lens bend in a meniscus shape at the early stages of the design, this constraint had to be released. Another mechanical constraint imposed was a positive back focal length.

In order to achieve the desired optical performance, both elements 1 and 2 now include aspherical surfaces. The positive focusing group in the original design is substituted by a negative doublet that controls color and distortion but also increases back focal length.

The resulting system has the desired focal length but $60 \mathrm{~mm}$ total track, that is, 0.075 telephoto ratio with $13 \%$ obstruction and $4 \mathrm{~mm}$ image clearance. The image quality is diffraction limit on axis but not for off axis fields due to the chromatic aberration. Distortion is virtually zero.

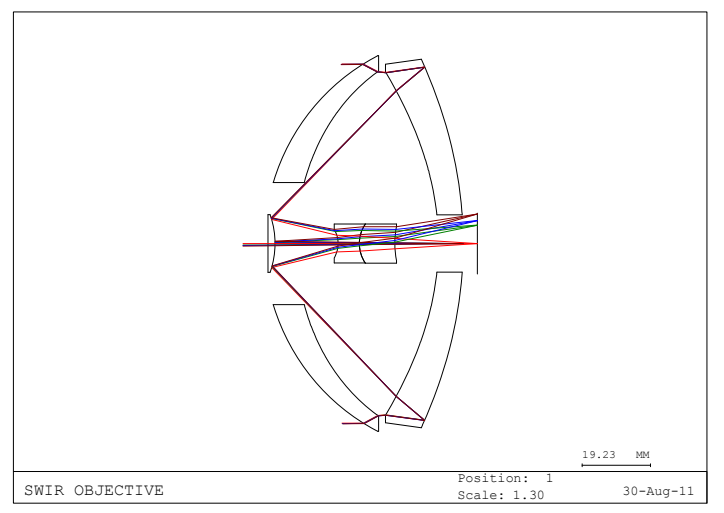

(a)

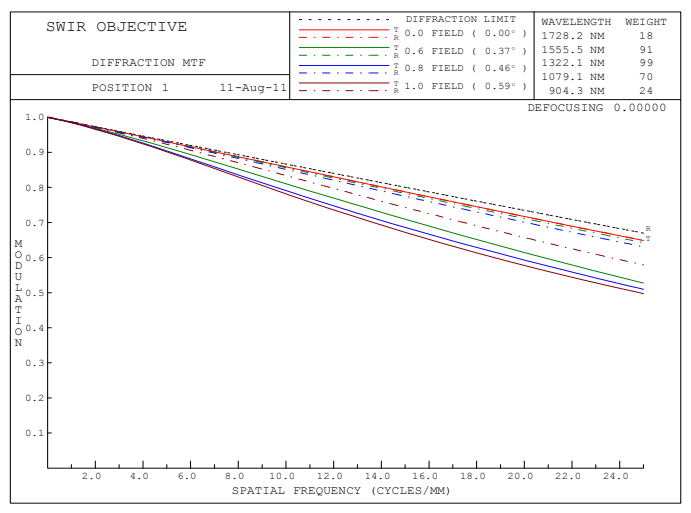

(b)

Figure 2: SWIR objective (a) Lens configuration (b) Image quality 


\subsection{Using an aplanatic design as a starting point}

Aplanatic optical system designs are free from on-axis spherical aberration and linear coma. These two conditions mean that (1) stigmatic on-axis image and (2) the abbe sine condition. The opposite is also true, a design which produces a stigmatic on-axis image and fulfilling the Abbe sine condition is aplanatic.

Considering a Cassegrain configuration, the aplanatic condition is achieved with two mirrors whose curvatures are designed using two conditions, the Abbe sine conditions and the Fermat principle. The design procedure of an aplanatic system with two surfaces is described in [3]. Of course, the optical system may have more than two surfaces. In general it is possible to design an aplanatic system by designing only two of them, i.e., considering all other surfaces prescribed or there is another possibility which we will use to design the telephoto. These other surfaces can be used for different purposes. In this design we will add additional elements during the optimization process so we will consider the aplanatic design as a starting point.

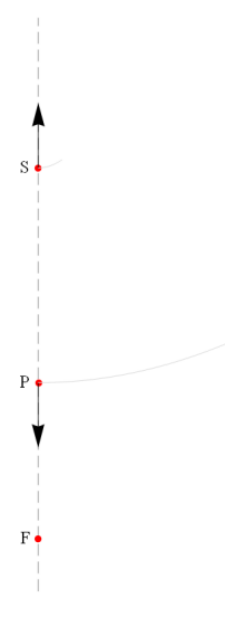

(a)

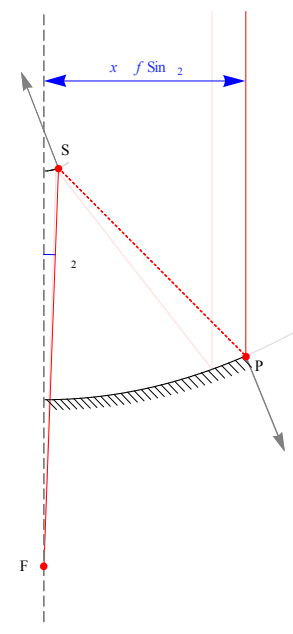

(b)

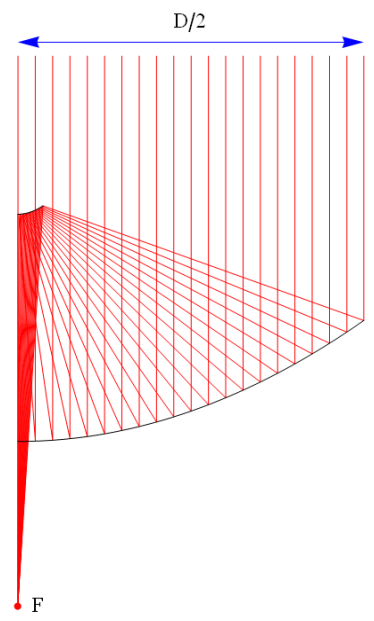

(c)

Figure 3. Design process of two aplanatic mirrors (a) Initial points and normals of the design (b) the rays from the point $\mathrm{F}$ with a certain $\theta$ after two reflections must go to a point with a coordinate $\mathrm{x}$ such that $x=f \sin \theta$ (c) The final design designed for the half aperture $(\mathrm{D} / 2)$ 
Note that the method gives us a list of points and normals for each mirror so they must be approximated by some function. There are a lot of different functions to approximate the mirrors however it is quite useful to approximate them using Qcon polynomials [4] because of the benefits that they have.

Once rotationally symmetric surfaces are approximated by a Qcon polynomial, they are exported to an optical design software, CODEV ${ }^{\circledR}$ in this case.

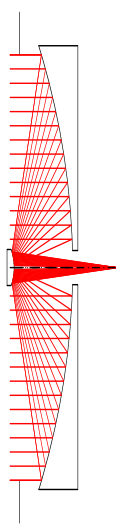

(a)

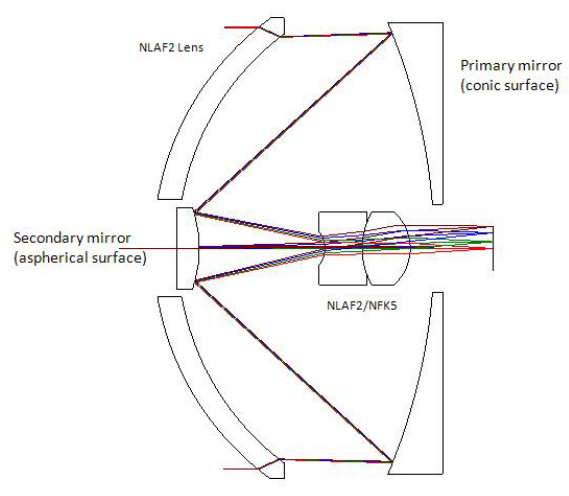

(b)

Figure 4 Designing a starting point from an aplanatic system (a) starting point for the optimization process (b) Final design.

As the design was started from an aplanatic design, only on-axis perfect image quality is achieved. In order to correct the field aberrations and distortion, more elements must be added to the system. For this design we decided to use a doublet in order to correct the chromatic and help with the field aberrations. Distortion is corrected by a front lens with second surface aspherical.

As compared with the previous system, this one is slightly larger: $62 \mathrm{~mm}$ but obstruction is much reduced, down to $4.8 \%$. Off-axis image quality is improved and distortion is about $1 \%$ as shown in the next figure.

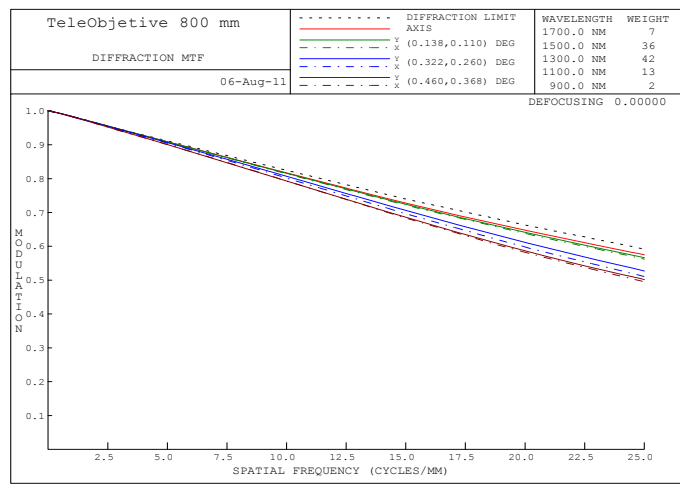

(a)

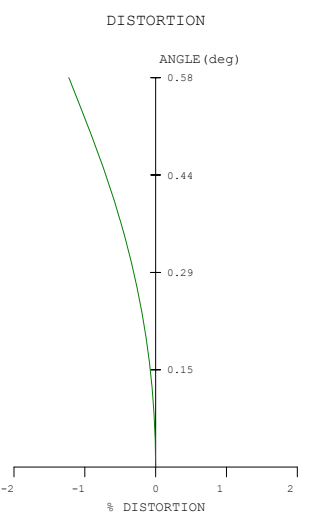

(b)

Figure 5 SWIR objective (a) MTF image quality (b) Distortion. 


\subsection{Using the SMS2D method}

As we have seen in the previous design it is possible to obtain good results using an aplanatic design as a starting point. Considering that the aplanatic design is a particular case of two surface SMS2D designs [6], [14][19], the next natural step would be to use the SMS2D method in order to obtain another starting point closer to the final result.

The standard SMS2D process includes two parts: Initial curves selection and Recursive Cartesian oval calculation [7-14]. The initial curves selection plays an important role in the SMS calculation because all the curves will grow in small segments from selected initial curves. A good selection of initial curves differs greatly from a bad selection in both stability of the SMS process and performance of the final optical design. Since the central vertices of initial curves can be defined loosely from mechanical or optical requirements of the system, a set of criteria is needed for the selection of the central shape of the initial curves. From paraxial image calculation or aplanatic conditions, initial curves can only control axial rays, without considering non-axial rays

Here we propose a set of wavefront criteria for 3 initial curves selections, as shown in Fig 1 . The theory of the SMS method states that 3 surfaces can perfectly image 3 input wavefronts into 3 output wavefronts [8]. In this work, only meridian ray bundles are selected for the SMS2D design, therefore they are placed symmetrically about the optical axis, which is a reasonable representation of all the meridian ray bundles. Points $V_{1}, V_{2}, V_{3}$ are central vertices of the initial curves that can be defined beforehand by system requirements. $R_{2}$ is the chief ray which passes from an on-axis point $\mathrm{Obj}_{2}$ to an on-axis point $\operatorname{Img}_{2}$ along the optical axis. $\mathrm{R}_{1}$ is the chief ray which passes from an off-axis point $\mathrm{Obj}_{1}$ to an off-axis point $\mathrm{Img}_{1}$ and $\mathrm{R}_{3}$ is its symmetrical counterpart. $\mathrm{R}_{1}, \mathrm{R}_{2}$, $\mathrm{R}_{3}$ all pass through the vertex of middle curve: $V_{2}$.

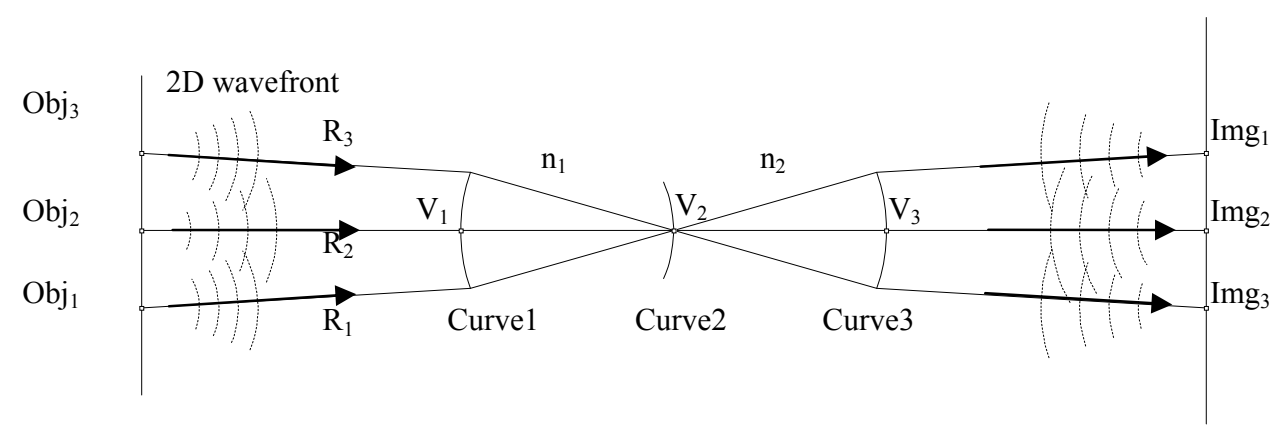

Figure 6. Wavefront criteria for initial curves selection

The wavefront criteria requires that the meridian wavefronts around the chief rays of 3 object points should be transformed into the meridian wavefronts of their corresponding image points by passing through this rotational optical system with generalized ray-tracing[15]. In this way, not only axial rays, but also non-axial meridian rays around $R_{1}$ and $R_{3}$ form perfect images by initial central curves. The solution to wavefront criteria might not be unique. It often results in several different shapes of initial curves. Later, after an SMS construction for each selection of initial curves, it can be determined which is the better solution, by simply raytracing designed raybundles (meridian ray-bundles in our case). The better solution to wavefront criteria should have better control of the designed rays.

Once the initial curves are selected, the rest of each curve can be grown from the initial curves, as shown in Figure 2. 

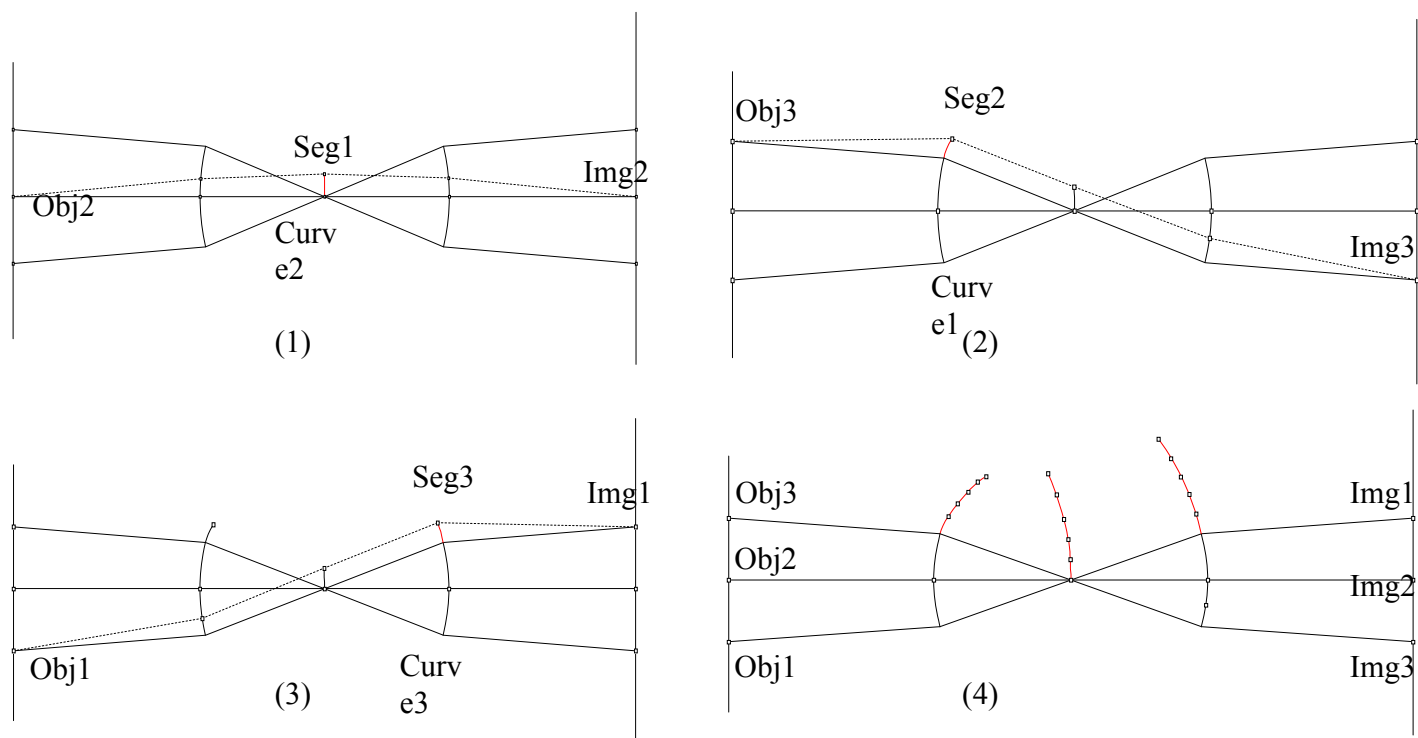

Figure 7. SMS growth process

Primarily, a small segment Seg1 of curve 2 is calculated as a Cartesian oval from the existing part of curve 1 and curve 3 to image $\mathrm{Obj}_{2}$ into $\mathrm{Img}_{2}$; secondarily, a small segment Seg2 of curve 1 is then calculated through Seg 1 and curve 3 to image $\mathrm{Obj}_{3}$ into $\mathrm{Img}_{3}$; thirdly, Seg3 of curve 3 can be calculated from Seg1 and curve 1 to image $\mathrm{Obj}_{1}$ into $\mathrm{Img}_{1}$. By repeating the same process, all segments can then be grown from the initial curves.

In order to achieve high compactness with high optical performance we suggest a XRXR SMS2D design. In SMS nomenclature $\mathrm{X}$ it is refers to a reflective surface and $\mathrm{R}$ for refractive surface.

In the design procedure described above, the first refractive surface is pre-given a certain curvature. This affords an additional freedom to optimize the optical system, because we have only designed the system with meridian rays, without controlling skew rays. The optimization is achieved by simply varying the central curvature of the first given parabola, and then continuing the SMS2D construction for the rest three curves, which is a onevariable optimization process. The merit function for this optimization is the largest RMS spot size, calculated by ray-tracing both meridian rays and skew rays.

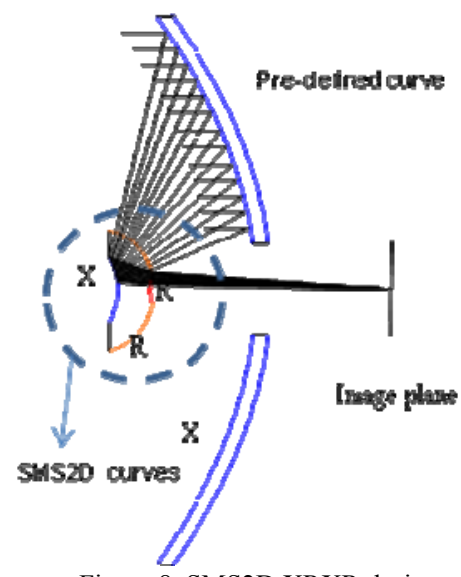

Figure 8. SMS2D XRXR design 
Using the SMS2D construction the resulting starting point is shown in figure 9. As can be seen, the monochromatic system is diffraction limit over most of the field of view. At this point only fictitious glass and for a single wavelength of $1300 \mathrm{~nm}$ have been used.

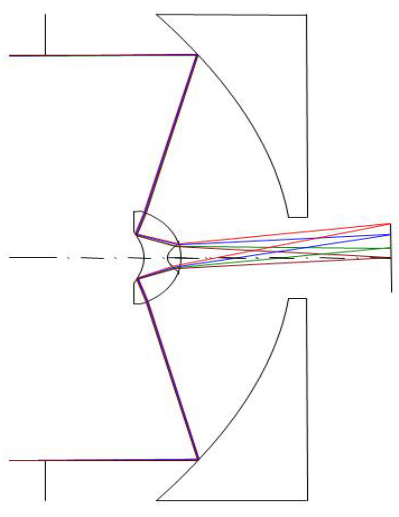

(a)

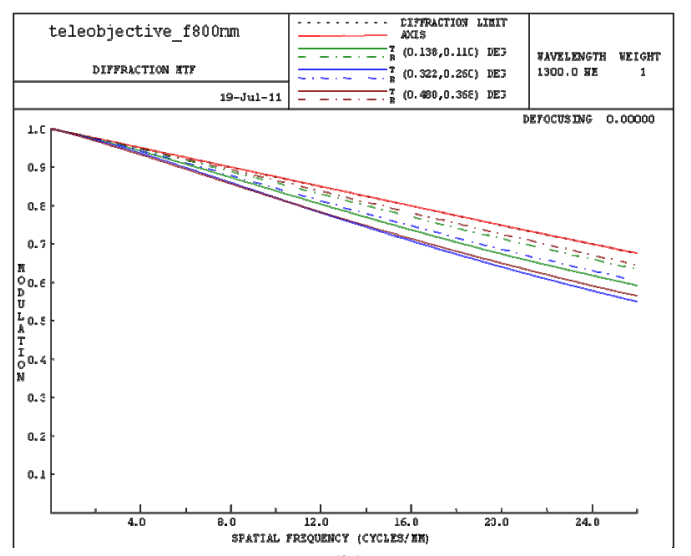

(b)

Figure 9. (a) Starting point given by the SMS2D (b) Image quality

Optimization process has an important role to control all the requirements that have not been covered during the SMS method as the color correction. As more degrees of freedom are needed, the strategy is to transform the element designed using the SMS method into a doublet and using a field lens to maintain control over off axis aberrations. The choice of the glass is automatic using the CODEV ${ }^{\circledR}$ macro "glass expert" which is quite useful specially when your starting point is monochromatic.

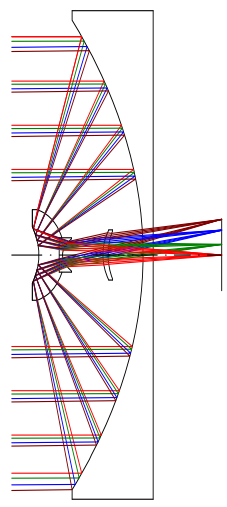

Figure 10.SWIR objective final design 


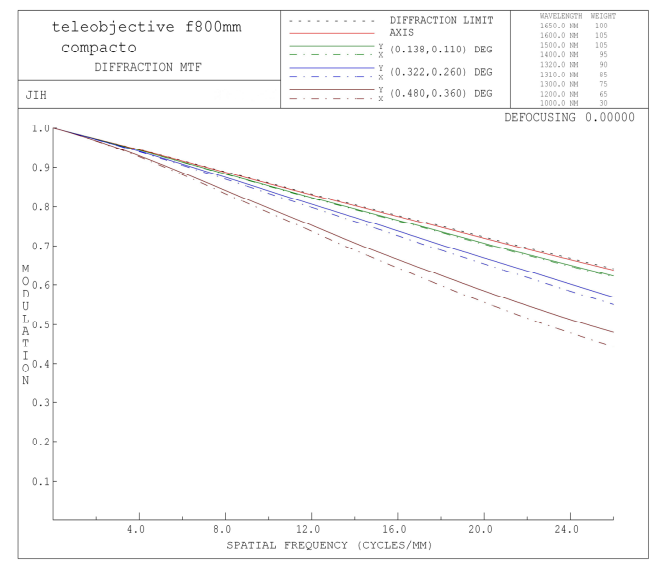

(a)

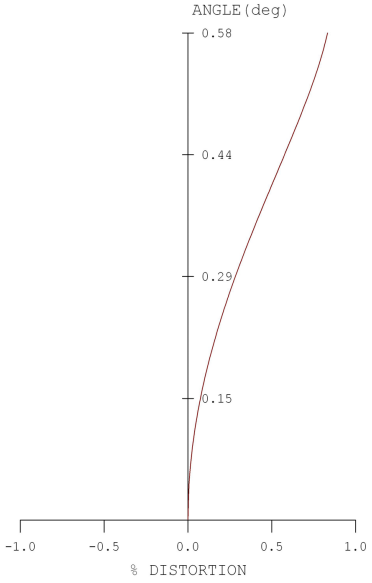

(b)

Figure 11. SWIR objective (a) Image quality. (b) Distortion.

Resulting system is the shortest one with a total track of only $52 \mathrm{~mm}$ (telephoto ratio 0.065 ) plus smallest obstruction, only $4.3 \%$. Besides, because the first element has been removed its weight has been reduced to only $210 \mathrm{gr}$ for an aluminium primary that is $39 \%$ of the weight for the first system.

\section{Conclusions}

In this paper we have focused more on the starting points than on the optimization process because our interest is to answer the best way to face a design. Although using a previous design can yield a good design, the optimization process and the merit function is more complicated than for other approaches. Starting from an aplanatic predesign, results in a very similar configuration, but unlike previous case the starting point is completely monochromatic due to the fact aplantic design process only considers one wavelength. For this reason the glass choice must be taken into account during the optimization process. Finally using with SMS method starting point is very close to the final design what makes optimization process simpler than for the others cases. Using a powerful tool as automatic process for the glass choice, the SMS provide us a good design with the same image quality with minimum volume and weight.

\section{ACKNOWLEDGEMENTS}

Authors thank the Spanish Ministries, SEM: TSI-020302-2010-65) and the Madrid Regional Government (SPIR: 50/2010O.23/12/09,TIC2010 and O-PRO: PIE/209/2010) and UPM (Q090935C59) for the support given in the preparation of the present work. The authors also thank to Synopsys (formerly Optical Research Associates) customer service for all their help.

\section{REFERENCES}

[1] Terrence S. Lomheim and E.D. Hernandez-Baquero, "Translation of spectral radiance levels, band choices, and signal-to-noise requirements to focal plane specifications and design constraints," Proc. SPIE 4486, pp. 263 (2002).

[2] Craig Olson,Tim Goodman, Chris Addiego, Steve Mifsud, "Design and construction of a short-wave infrared 3.3X continuos zoom lens" Proc SPIE 7652, (2010)

[3] Luneburg, R. K., [Mathematical Theory of Optics], University of California Press, Berkeley, (1964).

[4]G.W. Forbes, "Shape specification for axially symmetric optical surfaces", Opt. Exp. 15, 5218-5226, 2007. See thebasis for mild aspheres discussed in Sec. 3, and especially Eq.(16). 
[5] G.W. Forbes and C.P. Brophy, “Asphere, O Asphere, how shall we describe thee?”, Optical Systems Design, SPIE Proceedings 7100, (2008)

[6] Chapter 8 in R.Winston, J.C. Miñano, P. P. Benítez, with contributions of N. Shatz, J. Bortz, "Nonimaging Optics", Academic Press Elsevier (2004).

[7] Chaves, J., [Introduction to Nonimaging Optics], CRC Press, (2008).

[8] Pablo Benítez, Juan C. Miñano, José Blen, Rubén Mohedano, Júlio Chaves, Oliver Dross, Maikel Hernández, and Waqidi Falicoff, "Simultaneous multiple surface optical design method in three dimensions," Opt. Eng. 43, 1489 (2004).

[9] P. Benítez and J. C. Miñano, "Ultra high-numerical-aperture imaging concentrator," J. Opt. Soc. Am. A, Vol 14 (1997).

[10] J. C. Miñano, P. Benítez, Wang Lin, José Infante, Fernando Muñoz, Asunción Santamaría

"An application of the SMS method for imaging designs," Optical Express, Vol 17, No. 26, p. 24036 (2009).

[11] Miñano, J.C., Benítez, P., Wang Lin,, Muñoz, F., Infante, J., Santamaría, A., "Overview of the SMS design method applied to imaging optics", Proc. SPIE 7429, 74290C (2009).

[12] Jose M. Infante Herrero, Fernando Muñoz, Pablo Benitez, Juan Carlos Miñano, Wang Lin,

Juan Vilaplana, Guillermo Biot, Marta de la Fuente "Novel fast catadioptric objective with wide field of view", Proc. SPIE 7787, 778704 (2010).

[13] F. Muñoz, P. Benítez, and J. C. Miñano "High-order aspherics: the SMS nonimaging design method applied to imaging optics", Proc. SPIE 7061, , (2008).

[14] Fernando Muñoz, [Sistemas ópticos avanzados de gran compactibiliad con aplicaciones en formación de imagen y en iluminación], Doctoral Disertation, UPM, Madrid (2004)

[15] Orestes N. Stavroudis, [The mathematics of geometrical and physical optics], Wiley-VCH (2006). 